\title{
Minimal modifications to the Tri-Bimaximal neutrino mixing
}

\section{Zhen-hua Zhao}

Institute of High Energy Physics, Chinese Academy of Sciences, P.O. Box 918, Beijing, 100049 China

E-mail: zhaozhenhua@ihep.ac.cn

ABSTRACT: In light of the observation of a relatively large $\theta_{13}$, the ever popular TriBimaximal (TBM) neutrino mixing which predicts a vanishing $\theta_{13}$ needs modifications. In this paper, we shall discuss the possibility of modifying it in a minimal way to fulfil this task. In the first part, a neutrino mass matrix with three independent parameters, which leads to the TM2 mixing, is obtained by analogy with that for the TBM mixing. In the second part, a model that can realize the TM2 mixing is constructed with flavor symmetries $A_{4} \times \mathrm{U}(1) \times Z_{2} \times Z_{2} \times Z_{2}$. It is the variant of a model that gives the TBM mixing, with only one more flavon field included. Furthermore, the imaginary vacuum expectation value (VEV) of this flavon breaks the imposed CP symmetry and results in $\theta_{23}=45^{\circ}$ and the maximal $\mathrm{CP}$ violation. Besides, this model building approach can be generalized to the TM1 mixing in a straightforward way.

Keywords: Neutrino Physics, CP violation, Discrete and Finite Symmetries

ARXIV EPRINT: 1405.3022 


\section{Contents}

1 Introduction 1

2 A minimal modification to the mass matrix 2

3 A minimal modification to the model building 5

3.1 The VEV alignments 6

3.2 The mass matrix and mixing pattern 8

$\begin{array}{llr}3.3 & \text { Leptogenesis } & 10\end{array}$

$\begin{array}{lll}3.4 & \text { Discussions } & 12\end{array}$

$\begin{array}{llr}4 & \text { Summary } & 14\end{array}$

\section{Introduction}

The fact of neutrino oscillations has been established. On the theoretical side, it can be explained by neutrinos having masses, and well described by a $3 \times 3$ unitary matrix - the PMNS matrix $[1,2]$ plus two mass squared differences $\Delta m_{21}^{2}=m_{2}^{2}-m_{1}^{2}$ and $\Delta m_{31}^{2}=m_{3}^{2}-m_{1}^{2}$. The PMNS matrix can be parameterized by three mixing angles and three CP phases,

$$
U_{\mathrm{PMNS}}=\left(\begin{array}{ccc}
c_{12} c_{13} & s_{12} c_{13} & s_{13} e^{-i \delta} \\
-s_{12} c_{23}-c_{12} s_{23} s_{13} e^{i \delta} & c_{12} c_{23}-s_{12} s_{23} s_{13} e^{i \delta} & s_{23} c_{13} \\
s_{12} s_{23}-c_{12} c_{23} s_{13} e^{i \delta} & -c_{12} s_{23}-s_{12} c_{23} s_{13} e^{i \delta} & c_{23} c_{13}
\end{array}\right)\left(\begin{array}{cc}
e^{i \alpha} & \\
& e^{i \beta} \\
& \\
& 1
\end{array}\right),
$$

where $s_{i j}$ and $c_{i j}$ stand for $\sin \theta_{i j}$ and $\cos \theta_{i j}$. As in the CKM matrix [3, 4], there is a Dirac CP-violating phase $\delta$. Differently, two Majorana phases $\alpha$ and $\beta$ may appear or not, depending on the nature of neutrino masses. In this paper, neutrino masses will be taken as Majorana ones. On the experimental side, neutrinos from different sources, ranging from the sun [5] and the atmosphere [6] to reactors [7] and accelerators [8], have been observed to oscillate among different flavors. Thanks to the accumulation of data, neutrino mixing parameters have been measured with a high precision. According to the latest global-fit results [9], they have the values as given in table 1 . Only the values in the case of normal hierarchy are shown here, because the models in this paper just allow this situation.

Before the measurement of $\theta_{13}[7]$, the TBM mixing $[10,11]$ was very popular,

$$
U_{\mathrm{TBM}}=\left(\begin{array}{ccc}
\frac{\sqrt{2}}{\sqrt{3}} & \frac{1}{\sqrt{3}} & 0 \\
-\frac{1}{\sqrt{6}} & \frac{1}{\sqrt{3}} & \frac{1}{\sqrt{2}} \\
\frac{1}{\sqrt{6}} & -\frac{1}{\sqrt{3}} & \frac{1}{\sqrt{2}}
\end{array}\right),
$$




\begin{tabular}{|c|c|c|c|c|}
\hline Parameter & best fit & $1 \sigma$ range & $2 \sigma$ range & $3 \sigma$ range \\
\hline $\sin ^{2} \theta_{12} / 10^{-1}$ & 3.23 & $3.07-3.39$ & $2.92-3.57$ & $2.78-3.75$ \\
\hline $\sin ^{2} \theta_{13} / 10^{-2}$ & 2.34 & $2.14-2.54$ & $1.95-2.74$ & $1.77-2.94$ \\
\hline $\sin ^{2} \theta_{23} / 10^{-1}$ & 5.67 & $4.39-5.99$ & $4.13-6.23$ & $3.92-6.43$ \\
\hline$\Delta m_{21}^{2}\left[10^{-5} \mathrm{eV}^{2}\right]$ & 7.60 & $7.42-7.79$ & $7.26-7.99$ & $7.11-8.18$ \\
\hline$\left|\Delta m_{31}^{2}\right|\left[10^{-3} \mathrm{eV}^{2}\right]$ & 2.48 & $2.41-2.53$ & $2.35-2.59$ & $2.30-2.65$ \\
\hline$\delta / \pi$ & 1.34 & $0.96-1.98$ & $0.00-2.00$ & $0.00-2.00$ \\
\hline
\end{tabular}

Table 1. Global-fit results for neutrino oscillation parameters.

whose prediction for mixing angles

$$
\sin ^{2} \theta_{12}=\frac{1}{3}, \quad \sin ^{2} \theta_{23}=\frac{1}{2}, \quad \theta_{13}=0,
$$

was in good agreement with experimental results at that time. When the charged leptons are diagonal, a neutrino mass matrix of the following form can give us the TBM mixing,

$$
M_{\nu}=\left(\begin{array}{lll}
A & & \\
& A & \\
& & A
\end{array}\right)+\left(\begin{array}{ccc}
0 & C & -C \\
C & B+C & B \\
-C & B & B+C
\end{array}\right) .
$$

Due to its simplicity and predictive power, many models starting from a discrete nonAbelian flavor symmetry [12-14] were proposed to realize this mass matrix and thus the TBM mixing.

However, considering the significant deviation of $\theta_{13}$ from 0 , we need to modify the TBM mixing [15-27]. First of all, a natural question arises as whether there is still a neutrino mass matrix that can accommodate the large $\theta_{13}$ and assumes a simple form like that in eq. (1.4) [28-30]. In section 2, a mass matrix for this purpose is obtained through twisting eq. (1.4), and its phenomenological consequences are discussed. As we will see, this mass matrix actually leads to the so-called TM2 mixing [15]. Therefore, a model with flavor symmetries is constructed to realize this mixing pattern in section 3. Special attention will be paid to the origin of $\mathrm{CP}$ violation. Furthermore, the generalization of this model to the TM1 mixing is also discussed $[15,31]$. Finally, a brief summary is given in section 4 .

\section{A minimal modification to the mass matrix}

In this section, we will modify eq. (1.4) minimally to produce a realistic neutrino mixing pattern. The mass matrix given below can take this responsibility,

$$
M_{\nu}=\left(\begin{array}{ccc}
a & & \\
& a & \\
& & a
\end{array}\right)+\left(\begin{array}{ccc}
0 & c & c \\
c & b-c & b \\
c & b & b+c
\end{array}\right) .
$$


It can be viewed as a sister matrix of eq. (1.4), in the sense that their elements possess similar relations,

$$
m_{\mu \mu}-m_{e e}=m_{\mu \tau}-m_{e \tau}, \quad m_{\tau \tau}-m_{e e}=m_{\mu \tau}+m_{e \mu}, \quad m_{e \mu}= \pm m_{e \tau}
$$

The only difference lies in the fact that eq. (1.4) takes $m_{e \mu}=-m_{e \tau}$, while eq. (2.1) takes $m_{e \mu}=m_{e \tau}$. Thus, the latter case can be taken as a minimal modification to the former case. The above way of obtaining eq. (2.1) is a little novel and seems not to be reasonable. However, it can also be reached from other perspectives which have solid ground. First of all, we notice that it has something to do with the Friedberg-Lee symmetry [32-35] which shapes the neutrino mass matrix to be as

$$
M_{\nu}=\left(\begin{array}{ccc}
a & & \\
& a & \\
& & a
\end{array}\right)+\left(\begin{array}{ccc}
c+d & -d & c \\
-d & b+d & b \\
c & b & b+d
\end{array}\right) .
$$

By choosing $d=-c$, this equation can be reduced to eq. (2.1), so the latter has one parameter fewer and its results are more predictive as we will see. Eq. (2.1) can also be understood in terms of the $\mu-\tau$ symmetry [36] and its breaking [37-44]. As pointed out in ref. [45], a general neutrino mass matrix can be decomposed into two parts,

$$
M_{\nu}=\left(\begin{array}{ccc}
M_{e e} & M_{e \mu}^{+} & -M_{e \mu}^{+} \\
M_{e \mu}^{+} & M_{\mu \mu}^{+} & M_{\mu \tau} \\
-M_{e \mu}^{+} & M_{\mu \tau} & M_{\mu \mu}^{+}
\end{array}\right)+\left(\begin{array}{ccc}
0 & M_{e \mu}^{-} & M_{e \mu}^{-} \\
M_{e \mu}^{-} & M_{\mu \mu}^{-} & 0 \\
M_{e \mu}^{-} & 0 & -M_{\mu \mu}^{-}
\end{array}\right),
$$

where the first part obeys the $\mu-\tau$ symmetry, while the second part breaks it. In eq. (2.1), $a$ and $c$ obey the $\mu-\tau$ symmetry, while $b$ which corresponds to taking $M_{\mu \mu}^{-}=-M_{e \mu}^{-}$violates it. Anyway, we can put aside the origin of eq. (2.1) for the time being and just study its implications for phenomenology.

In order to make physical results manifest, instead of the standard parametrization in eq. (1.1), the matrix that diagonalizes eq. (2.1) is parameterized in a different way,

$$
U_{\nu}=R\left(\theta_{23}^{\prime}\right) R\left(\theta_{12}^{\prime}\right) R\left(\theta_{13}^{\prime}\right)
$$

where $R\left(\theta_{i j}^{\prime}\right)$ is a rotation in the $i-j$ plane by the angle $\theta_{i j}^{\prime}$. Here the superscript " $"$ " is used to distinguish the angles from those in the standard parametrization. In this case, the three angles can be expressed as

$$
\sin \theta_{23}^{\prime}=\frac{1}{\sqrt{2}}, \quad \sin \theta_{12}^{\prime}=\frac{1}{\sqrt{3}}, \quad \sin \theta_{13}^{\prime}=\frac{\sqrt{b^{2}+3 c^{2}}-b^{2}}{\sqrt{2\left(b^{2}+3 c^{2}\right)-2 b^{2} \sqrt{b^{2}+3 c^{2}}}},
$$

while the mass eigenvalues are

$$
m_{1}=a+b-\sqrt{b^{2}+3 c^{2}}, \quad m_{2}=a, \quad m_{3}=a+b+\sqrt{b^{2}+3 c^{2}} .
$$


In the basis where the charged leptons are diagonal, $U_{\mathrm{PMNS}}$ is identical with $U_{\nu}$,

$$
U_{\mathrm{PMNS}}=\left(\begin{array}{ccc}
\frac{\sqrt{2}}{\sqrt{3}} & \frac{1}{\sqrt{3}} & 0 \\
-\frac{1}{\sqrt{6}} & \frac{1}{\sqrt{3}} & \frac{1}{\sqrt{2}} \\
\frac{1}{\sqrt{6}} & -\frac{1}{\sqrt{3}} & \frac{1}{\sqrt{2}}
\end{array}\right)\left(\begin{array}{cc}
\cos \theta_{13}^{\prime} & \sin \theta_{13}^{\prime} \\
& 1 \\
-\sin \theta_{13}^{\prime} & \cos \theta_{13}^{\prime}
\end{array}\right) .
$$

Confronting eq. (2.8) with eq. (1.1), neutrino mixing angles in the standard parameterization can be extracted as follows,

$$
\sin \theta_{13}=\frac{\sqrt{2}}{\sqrt{3}} \sin \theta_{13}^{\prime}, \quad \sin \theta_{12}=\frac{\frac{1}{\sqrt{3}}}{\sqrt{1-\frac{2}{3} \sin ^{2} \theta_{13}^{\prime}}}, \quad \sin \theta_{23}=\frac{\left(-\frac{1}{\sqrt{6}} \sin \theta_{13}^{\prime}+\frac{1}{\sqrt{2}} \cos \theta_{13}^{\prime}\right)}{\sqrt{1-\frac{2}{3} \sin ^{2} \theta_{13}^{\prime}}} .
$$

For definiteness, $\theta_{13}^{\prime}$ will take the following value which gives $\sin \theta_{13}=0.15$,

$$
\sin \theta_{13}^{\prime}=0.19, \quad \text { when } \quad \frac{c}{b}=\frac{2}{5 \sqrt{3}} .
$$

With this choice, the mixing angles can be calculated directly,

$$
\sin ^{2} \theta_{13}=0.0237, \quad \sin ^{2} \theta_{12}=0.341, \quad \sin ^{2} \theta_{23}=0.390 .
$$

Furthermore, the values of $a, b$ and $c$ are completely determined,

$$
a=5.06 \times 10^{-2} \mathrm{eV}, \quad b=9.81 \times 10^{-3} \mathrm{eV}, \quad c=2.27 \times 10^{-3} \mathrm{eV} .
$$

As a result, neutrino masses are calculable and they are of the normal hierarchy

$$
m_{1}=4.98 \times 10^{-2} \mathrm{eV}, \quad m_{2}=5.06 \times 10^{-2} \mathrm{eV}, \quad m_{3}=7.10 \times 10^{-2} \mathrm{eV} .
$$

$m_{\beta \beta}$ which regulates the rate of neutrino-less double beta decay and $\sum m_{i}$ can be obtained as

$$
m_{\beta \beta}=0.051 \mathrm{eV}, \quad \sum m_{i}=0.171 \mathrm{eV}
$$

which are very close to the experimental upper bounds, so expected to be observable in the near future.

The result for $\theta_{23}$ is on the edge of the $3 \sigma$ range of the global-fit results and outside of the $2 \sigma$ range of T2K's recent result $\sin ^{2} \theta_{23}=0.514_{-0.056}^{+0.055}$ [46]. However, it is consistent with results of the MINOS experiment $\sin ^{2} \theta_{23}=0.388_{-0.035}^{+0.051}[8]$ or $\sin ^{2} \theta_{23}=0.35-0.65$ (90\% C.L.) in another analysis [47]. Thus, we can't come to a definite conclusion before $\theta_{23}$ is measured with a high precision. More importantly, the prediction for $\theta_{23}$ will be changed if $\mathrm{CP}$ violation is taken into consideration. For example, we can take $c$ as a complex parameter $|c| e^{i \phi}$ while keeping $a$ and $b$ real, for which case the PMNS matrix becomes,

$$
U_{\mathrm{PMNS}}=\left(\begin{array}{ccc}
\frac{\sqrt{2}}{\sqrt{3}} & \frac{1}{\sqrt{3}} & 0 \\
-\frac{1}{\sqrt{6}} & \frac{1}{\sqrt{3}} & \frac{1}{\sqrt{2}} \\
\frac{1}{\sqrt{6}} & -\frac{1}{\sqrt{3}} & \frac{1}{\sqrt{2}}
\end{array}\right)\left(\begin{array}{ccc}
\cos \theta_{13}^{\prime} & & \sin \theta_{13}^{\prime} e^{-i \rho} \\
& 1 & \\
-\sin \theta_{13}^{\prime} e^{i \rho} & \cos \theta_{13}^{\prime}
\end{array}\right)
$$


where $\theta_{13}^{\prime}$ and $\rho$ can be obtained from

$$
\tan \rho=\frac{b \tan \phi}{a+b}, \quad \tan 2 \theta_{13}^{\prime}=\frac{\sqrt{3} c \cos \phi}{b \cos \rho} .
$$

Accordingly, there is a correlation among $\theta_{13}, \theta_{23}$ and $\rho$

$$
\sin ^{2} \theta_{23}=\frac{\frac{1}{2}\left(1-\sin ^{2} \theta_{13}\right)-\frac{1}{\sqrt{3}} \cos \rho \sin \theta_{13} \sqrt{\frac{3}{2}\left(1-\frac{3}{2} \sin ^{2} \theta_{13}\right)}}{1-\sin ^{2} \theta_{13}} .
$$

For illustration, we can fix $\sin \theta_{13}$ at 0.15 , then $\sin ^{2} \theta_{23}$ would vary from 0.393 to 0.607 when $\rho$ takes values in the range $[0,2 \pi]$. Obviously, we can go back to the mixing matrix given in eq. (2.8) by taking $\rho=0$. On the other hand, in the case of $\rho=\pi / 2$ or $3 \pi / 2$, $\theta_{23}$ remains maximal. This interesting possibility [48-50] is still allowed by experimental results and provides a promising CP-violating effect, with the Jarlskog invariant [51] as large as 0.036 .

\section{A minimal modification to the model building}

As we have seen, the modified mass matrix eq. (2.1) results in the TM2 mixing given by eq. (2.15) where the particular case $\rho=\pi / 2$ or $3 \pi / 2$ deserves special attention. A model realizing this mixing pattern will be given in the following. There have already been several models for this purpose in the literature $[52-57,59,60]$. But from a different point of view, we will achieve this goal by modifying a model that gives the TBM mixing as minimally as possible. Our starting point is an observation: as eq. (2.15) itself suggests, the PMNS matrix can be split into two parts which have different origins. This can be realized through the following thread: at the first stage, right handed neutrinos $N_{1}, N_{2}$ and $N_{3}$ are diagonal and their Yukawa couplings with left-handed neutrinos have such a form that light neutrinos have the TBM mixing after the seesaw mechanism [61-64]. At the second stage, a flavon field which acquires a VEV induces the mixing between $N_{1}$ and $N_{3}$, contributing the second part of the mixing matrix. In order to control the source of $\mathrm{CP}$ violation, we will impose the CP symmetry [65-70] and spontaneously break it by this same flavon field.

The model is constructed under the simplest non-Abelian discrete group $-A 4$, which has 4 different representations $\mathbf{1}, \mathbf{1}^{\prime}, \mathbf{1}^{\prime \prime}$ and $\mathbf{3}$, whose multiplication rules are listed here for consultation [58],

$$
\begin{aligned}
1^{\prime} \times 1^{\prime \prime} & \rightarrow & 1 & =a b \\
1^{\prime} \times 3 & \rightarrow & 3 & =\left(a b_{3}, a b_{1}, a b_{2}\right) \\
1^{\prime \prime} \times 3 & \rightarrow & 3 & =\left(a b_{2}, a b_{3}, a b_{1}\right) \\
3 \times 3 & \rightarrow & 1 & =a_{1} b_{1}+a_{2} b_{3}+a_{3} b_{2} \\
3 \times 3 & \rightarrow & 1^{\prime} & =a_{3} b_{3}+a_{1} b_{2}+a_{2} b_{1} \\
3 \times 3 & \rightarrow & 1^{\prime \prime} & =a_{2} b_{2}+a_{1} b_{3}+a_{3} b_{1} \\
3 \times 3 & \rightarrow & 3_{A} & =\left(a_{2} b_{3}-a_{3} b_{2}, a_{1} b_{2}-a_{2} b_{1}, a_{1} b_{3}-a_{3} b_{1}\right)
\end{aligned}
$$




\begin{tabular}{|c|c|c|c|c|c|c|c|c|c|c|c|c|c|}
\hline & $L_{i}$ & $e^{c}$ & $\mu^{c}$ & $\tau^{c}$ & $N_{1}$ & $N_{2}$ & $N_{3}$ & $H_{\mathrm{u}, \mathrm{d}}$ & $\phi$ & $\varphi$ & $\chi$ & $\psi$ & $\xi$ \\
\hline$A 4$ & 3 & 1 & $1^{\prime \prime}$ & $1^{\prime}$ & 1 & 1 & 1 & 1 & 3 & 3 & 3 & 3 & 1 \\
\hline$U 1$ & 1 & -5 & -3 & -2 & 0 & 0 & 0 & 0 & 1 & -1 & -1 & -1 & 0 \\
\hline$Z_{2}^{1}$ & 1 & 1 & 1 & 1 & -1 & 1 & 1 & 1 & 1 & -1 & 1 & 1 & -1 \\
\hline$Z_{2}^{2}$ & 1 & 1 & 1 & 1 & 1 & -1 & 1 & 1 & 1 & 1 & -1 & 1 & 1 \\
\hline$Z_{2}^{3}$ & 1 & 1 & 1 & 1 & 1 & 1 & -1 & 1 & 1 & 1 & 1 & -1 & -1 \\
\hline
\end{tabular}

Table 2. Quantum numbers of the fields.

and

$$
3 \times 3 \rightarrow 3_{S}=\left(2 a_{1} b_{1}-a_{2} b_{3}-a_{3} b_{2}, 2 a_{3} b_{3}-a_{1} b_{2}-a_{2} b_{1}, 2 a_{2} b_{2}-a_{1} b_{3}-a_{3} b_{1}\right) .
$$

$a_{i}$ and $b_{i}$ denote the components of a multi-dimensional representation. In order to establish the relations among different mass matrix elements as suggested by eq. (2.2), three lepton doublets $L_{i=1,2,3}$ are organized to form the representation 3. Since there are large hierarchies among the charged leptons, $e^{c}, \mu^{c}$ and $\tau^{c}$ (here we have employed the convention in supersymmetry (SUSY) to denote the singlets under the $\mathrm{SU}(2)_{\mathrm{L}}$ gauge symmetry) are specified as representations $\mathbf{1}, \mathbf{1}^{\prime \prime}$ and $\mathbf{1}^{\prime}$ respectively. An additional $\mathrm{U}(1)$ symmetry, which plays the same role as the well-known Froggatt-Nielsen symmetry [71], is introduced to produce these hierarchies, by letting $e^{c}, \mu^{c}$ and $\tau^{c}$ have different charges under it. As mentioned, we want the mass matrix for right-handed neutrinos to be diagonal at the first step, so they are arranged to be the representation 1 and have $Z_{2}^{i=1,2,3}$ quantum numbers respectively. The flavon field $\xi$ which is charged under both $Z_{2}^{1}$ and $Z_{2}^{3}$ will induce the mixing between $N_{1}$ and $N_{3}$ after obtaining a VEV. Finally, there are some other flavon fields $\phi, \varphi, \chi$ and $\psi$ which will spontaneously break the $A 4$ symmetry. All the fields and their quantum numbers are summarized in table 2 .

\subsection{The VEV alignments}

In models with discrete flavor symmetries, flavon fields such as $\phi, \varphi, \chi$ and $\psi$ which are multi-dimensional representations are normally required to have VEVs with specific alignments, so that a particular mixing pattern can be guaranteed. This model is not an exception and the VEVs have a form as follows

$$
\langle\phi\rangle=(1,0,0) V_{1}, \quad\langle\varphi\rangle=(2,-1,-1) V_{2}, \quad\langle\chi\rangle=(1,1,1) V_{3}, \quad\langle\psi\rangle=(0,1,-1) V_{4} .
$$

As usual, the reasonableness of this choice can be justified by the approach developed in [13]: in the framework of SUSY, we can introduce some "driving fields" to make the flavon fields have the required VEVs, with the help of R-symmetry - $\mathrm{U}(1)_{\mathrm{R}}$. Driving fields are the ones that have charge 2 under $U(1)_{R}$, while the flavon fields have charge 0 . Since the terms in superpotential are required to have charge 2 in total, they should have the form $\Delta(\cdots)$, where $\Delta$ represents a driving field and dots in the bracket are linear combinations of the flavon fields. If there are driving fields with quantum numbers as shown in table 3 , 


\begin{tabular}{|c|c|c|c|c|c|c|c|c|c|c|c|c|}
\hline & $\Delta_{1}$ & $\Delta_{2}$ & $\Delta_{3}$ & $\Delta_{4}$ & $\Delta_{5}$ & $\Delta_{6}$ & $\Delta_{7}$ & $\Delta_{8}$ & $\Delta_{9}$ & $\Delta_{10}$ & $\Delta_{11}$ & $\Delta_{12}$ \\
\hline$A 4$ & $1^{\prime}$ & $1^{\prime \prime}$ & 1 & 1 & 1 & 1 & $1^{\prime}$ & $1^{\prime \prime}$ & 1 & 1 & $1^{\prime}$ & $1^{\prime \prime}$ \\
\hline$U 1$ & -2 & -2 & -1 & 0 & 2 & 2 & 2 & 2 & 2 & 2 & 2 & 2 \\
\hline$Z_{2}^{1}$ & 1 & 1 & 1 & 1 & 1 & -1 & -1 & -1 & -1 & 1 & 1 & 1 \\
\hline$Z_{2}^{2}$ & 1 & 1 & 1 & 1 & 1 & -1 & -1 & -1 & 1 & -1 & -1 & -1 \\
\hline$Z_{2}^{3}$ & 1 & 1 & -1 & 1 & 1 & 1 & 1 & 1 & -1 & -1 & -1 & -1 \\
\hline
\end{tabular}

Table 3. Quantum numbers of Driving Fields.

the superpotential $-\mathcal{W}$, which is relevant to VEVs of the flavon fields, is constrained to the following form, up to next-to-leading-order (NLO),

$$
\begin{aligned}
& \Delta_{1}\left\{\lambda_{1}(\phi \phi)_{1^{\prime \prime}}\right\}+\Delta_{2}\left\{\lambda_{2}(\phi \phi)_{1^{\prime}}\right\}+\Delta_{3}\left\{\frac{\lambda_{3}}{\Lambda}(\phi \phi \psi)_{1}\right\}+\Delta_{4}\left\{\lambda_{4}(\xi \xi)_{1} \pm M^{2}\right\}+ \\
& \Delta_{5}\left\{\lambda_{5}(\varphi \varphi)_{1}+\lambda_{6}(\chi \chi)_{1}+\lambda_{7}(\psi \psi)_{1}+\frac{\lambda_{8}}{\Lambda}(\varphi \psi \xi)_{1}\right\}+\Delta_{6}\left\{\lambda_{9}(\varphi \chi)_{1}+\frac{\lambda_{10}}{\Lambda}(\chi \psi \xi)_{1}\right\}+ \\
& \Delta_{7}\left\{\lambda_{11}(\varphi \chi)_{1^{\prime \prime}}+\frac{\lambda_{12}}{\Lambda}(\chi \psi \xi)_{1^{\prime \prime}}\right\}+\Delta_{8}\left\{\lambda_{13}(\varphi \chi)_{1^{\prime}}+\frac{\lambda_{14}}{\Lambda}(\chi \psi \xi)_{1^{\prime}}\right\}+\Delta_{9}\left\{\lambda_{15}(\varphi \psi)_{1}+\right. \\
& \left.\frac{\lambda_{16}}{\Lambda}(\varphi \varphi \xi)_{1}+\frac{\lambda_{17}}{\Lambda}(\chi \chi \xi)_{1}+\frac{\lambda_{18}}{\Lambda}(\psi \psi \xi)_{1}\right\}+\Delta_{10}\left\{\lambda_{19}(\chi \psi)_{1}+\frac{\lambda_{20}}{\Lambda}(\varphi \chi \xi)_{1}\right\}+ \\
& \Delta_{11}\left\{\lambda_{21}(\chi \psi)_{1^{\prime \prime}}+\frac{\lambda_{22}}{\Lambda}(\varphi \chi \xi)_{1^{\prime \prime}}\right\}+\Delta_{12}+\left\{\lambda_{23}(\chi \psi)_{1^{\prime}}+\frac{\lambda_{24}}{\Lambda}(\varphi \chi \xi)_{1^{\prime}}\right\} .
\end{aligned}
$$

In the above, $\lambda_{i}$ are dimensionless coefficients and $M$ is a dimension-one parameter, and $\Lambda$ is the cut-off scale for non-renormalizable operators. The symbol $(\cdots)_{1 / 1^{\prime} / 1^{\prime \prime}}$ means that linear combinations in the bracket must form the representation $\mathbf{1}$ or $\mathbf{1}^{\prime}$ or $\mathbf{1}^{\prime \prime}$ to match the corresponding $\Delta_{i}$.

SUSY requires each $F$ component of the driving fields to have a vanishing VEV,

$$
\left\langle F_{i}^{*}\right\rangle=-\frac{\partial \mathcal{W}}{\partial \Delta_{i}}=0 .
$$

This leads to some constraint equations on the VEVs,

$$
\begin{aligned}
& \lambda_{1}\left(\phi_{2} \phi_{2}+2 \phi_{1} \phi_{3}\right)=0, \quad \lambda_{2}\left(\phi_{3} \phi_{3}+2 \phi_{1} \phi_{2}\right)=0, \\
& \lambda_{8}\left(\varphi_{1} \psi_{1}+\varphi_{2} \psi_{3}+\varphi_{3} \psi_{2}\right) \xi=0, \quad \lambda_{9}\left(\varphi_{1} \chi_{1}+\varphi_{2} \chi_{3}+\varphi_{3} \chi_{2}\right)=0, \\
& \lambda_{10}\left(\chi_{1} \psi_{1}+\chi_{2} \psi_{3}+\chi_{3} \psi_{2}\right) \xi=0, \quad \lambda_{11}\left(\varphi_{2} \chi_{2}+\varphi_{1} \chi_{3}+\varphi_{3} \chi_{1}\right)=0, \\
& \lambda_{12}\left(\chi_{2} \psi_{2}+\chi_{1} \psi_{3}+\chi_{3} \psi_{1}\right) \xi=0, \quad \lambda_{13}\left(\varphi_{3} \chi_{3}+\varphi_{1} \chi_{2}+\varphi_{2} \chi_{1}\right)=0, \\
& \lambda_{14}\left(\chi_{3} \psi_{3}+\chi_{1} \psi_{2}+\chi_{2} \psi_{1}\right) \xi=0, \quad \lambda_{15}\left(\varphi_{1} \psi_{1}+\varphi_{2} \psi_{3}+\varphi_{3} \psi_{2}\right)=0, \\
& \lambda_{19}\left(\chi_{1} \psi_{1}+\chi_{2} \psi_{3}+\chi_{3} \psi_{2}\right)=0, \quad \lambda_{20}\left(\varphi_{1} \chi_{1}+\varphi_{2} \chi_{3}+\varphi_{3} \chi_{2}\right) \xi=0, \\
& \lambda_{21}\left(\chi_{2} \psi_{2}+\chi_{1} \psi_{3}+\chi_{3} \psi_{1}\right)=0, \quad \lambda_{22}\left(\varphi_{2} \chi_{2}+\varphi_{1} \chi_{3}+\varphi_{3} \chi_{1}\right) \xi=0, \\
& \lambda_{23}\left(\chi_{3} \psi_{3}+\chi_{1} \psi_{2}+\chi_{2} \psi_{1}\right)=0, \quad \lambda_{24}\left(\varphi_{3} \chi_{3}+\varphi_{1} \chi_{2}+\varphi_{2} \chi_{1}\right) \xi=0,
\end{aligned}
$$


and

$$
\begin{aligned}
\lambda_{3}\left[\left(\phi_{1} \phi_{1}-\phi_{2} \phi_{3}\right) \psi_{1}+\left(\phi_{3} \phi_{3}-\phi_{1} \phi_{2}\right) \psi_{3}+\left(\phi_{2} \phi_{2}-\phi_{1} \phi_{3}\right) \psi_{2}\right] & =0 \\
\lambda_{5}\left(\varphi_{1} \varphi_{1}+2 \varphi_{2} \varphi_{3}\right)+\lambda_{6}\left(\chi_{1} \chi_{1}+2 \chi_{2} \chi_{3}\right)+\lambda_{7}\left(\psi_{1} \psi_{1}+2 \psi_{2} \psi_{3}\right) & =0 \\
\lambda_{16}\left(\varphi_{1} \varphi_{1}+2 \varphi_{2} \varphi_{3}\right) \xi+\lambda_{17}\left(\chi_{1} \chi_{1}+2 \chi_{2} \chi_{3}\right) \xi+\lambda_{18}\left(\psi_{1} \psi_{1}+2 \psi_{2} \psi_{3}\right) \xi & =0 .
\end{aligned}
$$

Eq. (3.3) is a solution to these equations, so it is fair to say that the VEVs can have the form as shown by it at least to NLO. Besides, there are some relations among $V_{2}, V_{3}$ and $V_{4}$,

$$
V_{3}=\sqrt{\frac{2 \lambda_{5} \lambda_{18}-2 \lambda_{7} \lambda_{16}}{\lambda_{7} \lambda_{17}-\lambda_{6} \lambda_{18}}} V_{2}, \quad V_{4}=\sqrt{\frac{3 \lambda_{5} \lambda_{17}-3 \lambda_{6} \lambda_{16}}{\lambda_{7} \lambda_{17}-\lambda_{6} \lambda_{18}}} V_{2} .
$$

In particular, $\xi$ also gets a VEV: $V_{5}= \pm M / \sqrt{\lambda_{4}}$ for the minus sign or $V_{5}= \pm i M / \sqrt{\lambda_{4}}$ for the plus sign in the below equation,

$$
\lambda_{4} \xi \xi \pm M^{2}=0 .
$$

The latter case will be the only source for $\mathrm{CP}$ violation in the lepton sector, if the $\mathrm{CP}$ symmetry is required. This method of obtaining a complex VEV for a scalar field is proposed in [72].

\subsection{The mass matrix and mixing pattern}

Now we can discuss the consequences of the model on mass matrices and the mixing pattern. The flavor symmetries only allow higher than dimension-four Yukawa-like operators, which have a general form $y_{i j} N_{i}^{c} L_{j} H_{\mathrm{u}}(\Phi / \Lambda)^{n_{i j}}$ or $y_{i j} E_{i}^{c} L_{j} H_{\mathrm{d}}(\Phi / \Lambda)^{n_{i j}}$. $\Phi$ represents the flavon fields $\phi, \varphi, \chi, \psi$ and $\omega$, and $\Lambda$ is the cut-off scale where an underlying theory emerges, when $n_{i j}$ is an integer measuring the power of $\Phi / \Lambda$. After the flavon fields gain VEVs which are commonly denoted as $V$, these operators become effective Yukawa terms $y_{i j} N_{i}^{c} L_{j} H_{\mathrm{u}}(V / \Lambda)^{n_{i j}}$ or $y_{i j} E_{i}^{c} L_{j} H_{\mathrm{d}}(V / \Lambda)^{n_{i j}}$. Usually, $V / \Lambda$ (labeled as $\epsilon$ ) is assumed to be an $\mathcal{O}(0.1)$ quantity. In this case, effective Yukawa couplings $y_{i j}(V / \Lambda)^{n_{i j}}$ are controlled by the corresponding coefficients $-\epsilon^{n_{i j}}$, so that mass hierarchies can be understood in terms of the power of $\epsilon$.

The terms that contribute to masses of the charged leptons include

$$
y_{1} \tau^{c} L H_{\mathrm{d}} \frac{\phi}{\Lambda}+y_{2} \mu^{c} L H_{\mathrm{d}} \frac{\phi^{2}}{\Lambda^{2}}+y_{3} e^{c} L H_{\mathrm{d}} \frac{\phi^{4}}{\Lambda^{4}},
$$

which lead to a diagonal mass matrix

$$
M_{\mathrm{l}}=\left(\begin{array}{ccc}
y_{3}\left(\frac{V_{1}}{\Lambda}\right)^{4} & & \\
& y_{2}\left(\frac{V_{1}}{\Lambda}\right)^{2} & \\
& & \\
& & y_{1} \frac{V_{1}}{\Lambda}
\end{array}\right) V_{\mathrm{d}} \sim\left(\begin{array}{ccc}
\epsilon_{1}^{4} & & \\
& \epsilon_{1}^{2} & \\
& & \epsilon_{1}
\end{array}\right) V_{\mathrm{d}},
$$

where $V_{\mathrm{d}}$ is the VEV of $H_{\mathrm{d}}$ and $V_{1} / \Lambda$ is replaced with $\epsilon_{1}$, indicating that it is a small quantity. Accordingly, the hierarchies of $m_{e}, m_{\mu}$ and $m_{\tau}$ get an explanation. On the other side, $Z_{2}^{i=1,2,3}$ symmetries fix the mass matrix for $N_{1}, N_{2}$ and $N_{3}$ to be diagonal too,

$$
M_{\mathrm{N}}=\left(\begin{array}{ccc}
M_{1} & & \\
& M_{2} & \\
& & M_{3}
\end{array}\right)
$$


which arises from the Majorana mass terms

$$
M_{1} N_{1}^{c} N_{1}^{c}+M_{2} N_{2}^{c} N_{2}^{c}+M_{3} N_{3}^{c} N_{3}^{c}
$$

The flavon fields $\varphi, \chi, \psi$ which have quantum numbers separately under $Z_{2}^{i=1,2,3}$ make the Yukawa couplings between left-handed and right-handed neutrinos have a form

$$
y_{4} N_{1}^{c} L H_{\mathrm{u}} \frac{\varphi}{\Lambda}+y_{5} N_{2}^{c} L H_{\mathrm{u}} \frac{\chi}{\Lambda}+y_{6} N_{3}^{c} L H_{\mathrm{u}} \frac{\psi}{\Lambda},
$$

which give the Dirac neutrino mass matrix as

$$
M_{\mathrm{D}}=\left(\begin{array}{ccc}
2 y_{4} & -y_{4} & -y_{4} \\
y_{5}^{\prime} & y_{5}^{\prime} & y_{5}^{\prime} \\
0 & -y_{6}^{\prime} & y_{6}^{\prime}
\end{array}\right) \epsilon_{2} V_{\mathrm{u}}
$$

where $V_{\mathrm{u}}$ is the VEV of $H_{\mathrm{u}}$ and $\epsilon_{2}$ is $V_{2} / \Lambda, y_{5}^{\prime}$ and $y_{6}^{\prime}$ are the abbreviations for $y_{5} \sqrt{\left(2 \lambda_{5} \lambda_{18}-2 \lambda_{7} \lambda_{16}\right) /\left(\lambda_{7} \lambda_{17}-\lambda_{6} \lambda_{18}\right)}$ and $y_{6} \sqrt{\left(3 \lambda_{5} \lambda_{17}-3 \lambda_{6} \lambda_{16}\right) /\left(\lambda_{7} \lambda_{17}-\lambda_{6} \lambda_{18}\right)}$.

The mass matrix for light neutrinos can be obtained through the seesaw mechanism,

$$
\begin{aligned}
M_{\nu}= & M_{\mathrm{D}}^{\mathrm{T}} M_{\mathrm{N}}^{-1} M_{\mathrm{D}} \\
= & \left(\begin{array}{ccc}
\frac{4 y_{4}^{2}}{M_{1}}+\frac{y_{5}^{\prime 2}}{M_{2}} & \frac{y_{5}^{\prime 2}}{M_{2}}-\frac{2 y_{4}^{2}}{M_{1}} & \frac{y_{5}^{\prime 2}}{M_{2}}-\frac{2 y_{4}^{2}}{M_{1}} \\
\cdots & \frac{y_{4}^{2}}{M_{1}}+\frac{y_{5}^{\prime 2}}{M_{2}}+\frac{y_{6}^{\prime 2}}{M_{3}} & \frac{y_{4}^{2}}{M_{1}}+\frac{y_{5}^{\prime 2}}{M_{2}}-\frac{y_{6}^{\prime 2}}{M_{3}} \\
\cdots & \cdots & \frac{y_{4}^{2}}{M_{1}}+\frac{y_{5}^{\prime 2}}{M_{2}}+\frac{y_{6}^{\prime 2}}{M_{3}}
\end{array}\right)\left(\epsilon_{2} V_{\mathrm{u}}\right)^{2},
\end{aligned}
$$

where the elements represented by "..." can be known through the symmetric property of $M_{\nu}$. It can be diagonalized by the TBM matrix

$$
U_{\mathrm{TBM}}^{\mathrm{T}} M_{\nu} U_{\mathrm{TBM}}=\left(\begin{array}{ccc}
\frac{6 y_{4}^{2}}{M_{1}} & & \\
& \frac{3 y_{5}^{\prime 2}}{M_{2}} & \\
& & \frac{2 y_{6}^{\prime 2}}{M_{3}}
\end{array}\right)\left(\epsilon_{2} V_{\mathrm{u}}\right)^{2} .
$$

Both of the normal and inverted hierarchies are allowed by this mass spectrum, but it's more natural for the former case. This is because the latter case needs a fine-tuning at one percent level to make the first two mass eigenvalues nearly degenerate, but $6 y_{4}^{2} / M_{1}$ and $3 y_{5}^{\prime 2} / M_{2}$ are two independent quantities. With the assumption $6 y_{4}^{2} \sim 3 y_{5}^{\prime 2} \sim 2 y_{6}^{\prime 2}$, the hierarchy between $\Delta m_{21}^{2}$ and $\Delta m_{31}^{2}$ can be attributed to the hierarchies among righthanded neutrinos $M_{1} \sim M_{2} \sim 5 M_{3}$. Up to now, we have reproduced the well-known TBM mixing. In the following, we will obtain the TM2 mixing by including the effect of $\xi$.

After $\xi$ obtains a VEV, the mass matrix for $N_{1}, N_{2}$ and $N_{3}$ becomes

$$
M_{\mathrm{N}}^{\prime}=\left(\begin{array}{ccc}
M_{1} & & \Delta M \\
& M_{2} & \\
\Delta M & & M_{3}
\end{array}\right),
$$


where $\Delta M=y V_{5}$ comes from the term $y N_{1} N_{3} \xi . M_{\mathrm{D}}$ remains the form given by eq. (3.15), so the mass matrix for light neutrinos turns into

$$
\begin{aligned}
& M_{\nu}^{\prime}=M_{\mathrm{D}}^{\mathrm{T}} M_{\mathrm{N}}^{\prime-1} M_{\mathrm{D}} \\
&=\left(\epsilon_{2} V_{\mathrm{u}}\right)^{2} \cdot\left(\begin{array}{ccc}
\frac{y_{5}^{\prime 2}}{M_{2}}+\frac{4 y_{4}^{2} M_{3}}{D} & \frac{y_{5}^{\prime 2}}{M_{2}}+\frac{2 y_{4}\left(y_{6}^{\prime} \Delta M-y_{4} M_{3}\right)}{D} & \frac{y_{5}^{\prime 2}}{M_{2}}-\frac{2 y_{4}\left(y_{6}^{\prime} \Delta M+y_{4} M_{3}\right)}{D} \\
\ldots & \frac{y_{5}^{\prime 2}}{M_{2}}+\frac{y_{6}^{\prime 2} M_{1}-2 y_{4} y_{6}^{\prime} \Delta M+y_{4}^{2} M_{3}}{D} & \frac{y_{5}^{\prime 2}}{M_{2}}-\frac{y_{6}^{\prime 2} M_{1}-y_{4}^{2} M_{3}}{D} \\
\ldots & \ldots & \frac{y_{5}^{\prime 2}}{M_{2}}+\frac{y_{6}^{\prime 2} M_{1}+2 y_{4} y_{6}^{\prime} \Delta M+y_{4}^{2} M_{3}}{D}
\end{array}\right),
\end{aligned}
$$

where $D=M_{1} M_{3}-(\Delta M)^{2}$. A TBM rotation transforms it into the following form

$$
U_{\mathrm{TBM}}^{\mathrm{T}} M_{\nu}^{\prime} U_{\mathrm{TBM}}=\left(\begin{array}{ccc}
\frac{6 y_{4}^{2} M_{3}}{D} & & -\frac{2 \sqrt{3} y_{4} y_{6}^{\prime} \Delta M}{D} \\
& \frac{3 y_{5}^{\prime 2}}{M_{2}} & \frac{2 y_{6}^{\prime 2} M_{1}}{D}
\end{array}\right)\left(\epsilon_{2} V_{\mathrm{u}}\right)^{2},
$$

which is then diagonalized by a rotation in the 1-3 plane,

$$
\mathrm{U}\left(\theta_{13}^{\prime}\right)=\left(\begin{array}{ccc}
\cos \theta_{13}^{\prime} & \sin \theta_{13}^{\prime} e^{-i \rho} \\
-\sin \theta_{13}^{\prime} e^{i \rho} & 1 & \cos \theta_{13}^{\prime}
\end{array}\right),
$$

with

$$
\begin{aligned}
& \rho=0, \quad \tan 2 \theta_{13}^{\prime}=\frac{2 \sqrt{3} y_{4} y_{6}^{\prime} \Delta M}{3 y_{4}^{2} M_{3}-y_{6}^{\prime 2} M_{1}}, \quad \text { if } \quad \mathrm{V}_{5}= \pm \frac{M}{\sqrt{\lambda_{4}}} \\
& \rho=\frac{3 \pi}{2} / \frac{\pi}{2}, \quad \tan 2 \theta_{13}^{\prime}=\frac{2 \sqrt{3} y_{4} y_{6}^{\prime}|\Delta M|}{3 y_{4}^{2} M_{3}+y_{6}^{\prime 2} M_{1}}, \quad \text { if } \quad \mathrm{V}_{5}= \pm i \frac{M}{\sqrt{\lambda_{4}}}
\end{aligned}
$$

As a result, the PMNS matrix $U_{\mathrm{PMNS}}=U_{\mathrm{TBM}} \mathrm{U}\left(\theta_{13}^{\prime}\right)$ has the same form as the TM2 mixing in three special cases: $\rho=0, \rho=\pi / 2$ or $\rho=3 \pi / 2$. As we have seen in eq. (2.10), $\theta_{13}^{\prime}$ should be about $1 / 5$ to generate the realistic $\theta_{13}$. This can be achieved by further assuming $\Delta M \sim 1 / 5 M_{1}$. In summary, the following approximation can be taken to fit the experimental results,

$$
6 y_{4}^{2} \sim 3 y_{5}^{\prime 2} \sim 2 y_{6}^{\prime 2}, \quad M_{1} \sim M_{2} \sim 5 M_{3} \sim 5|\Delta M| .
$$

\subsection{Leptogenesis}

Since the imaginary VEV of $\xi$ is the only source for CP violation in the lepton sector, it is also expected to play an important role in the leptogenesis mechanism [73], which is a popular scenario for generating the baryon asymmetry of the universe

$$
\frac{n_{\mathrm{B}}}{s}=(8.79 \pm 0.44) \times 10^{-11}
$$

Here $n_{\mathrm{B}}$ and $s$ are the number densities of baryons and entropy respectively. In this mechanism, the CP-violating and lepton-number-violating out-of-equilibrium decay of the 
lightest right-handed neutrino creates the net lepton number $\Delta L$, which is then converted to baryon number by the sphaleron process [74]. To study this issue, right-handed neutrinos are transformed to the mass basis $N_{1}^{\prime}, N_{2}^{\prime}$ and $N_{3}^{\prime}$ by a 1-3 rotation,

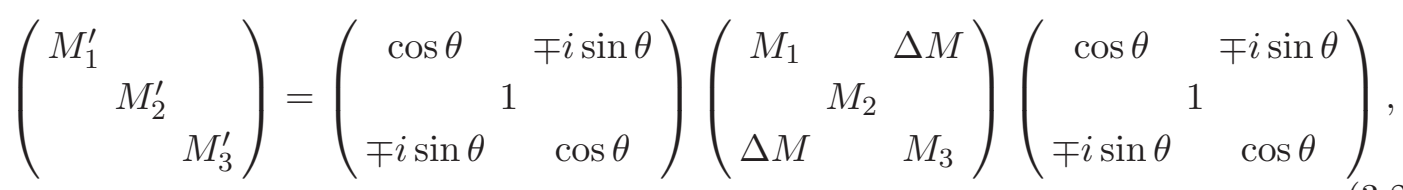

with $\tan 2 \theta=2|\Delta M| /\left(M_{1}+M_{3}\right)$. Correspondingly, the Yukawa couplings between $N_{i}^{\prime c}$ and $L_{j}$ are

$$
\eta=\left(\begin{array}{cc}
\cos \theta & \mp i \sin \theta \\
& 1 \\
\mp i \sin \theta & \cos \theta
\end{array}\right)\left(\begin{array}{ccc}
2 y_{4} & -y_{4} & -y_{4} \\
y_{5}^{\prime} & y_{5}^{\prime} & y_{5}^{\prime} \\
0 & -y_{6}^{\prime} & y_{6}^{\prime}
\end{array}\right) \epsilon_{2} .
$$

And $\eta \eta^{\dagger}$ which will be needed below has a form as

$$
\eta \eta^{\dagger}=\left(\begin{array}{cc}
6 y_{4}^{2} \cos ^{2} \theta+2 y_{6}^{\prime 2} \sin ^{2} \theta & i\left( \pm 6 y_{4}^{2} \mp 2 y_{6}^{\prime 2}\right) \cos \theta \sin \theta \\
i\left(\mp 6 y_{4}^{2} \pm 2 y_{6}^{\prime 2}\right) \cos \theta \sin \theta & 3 y_{5}^{\prime 2} \\
6 y_{4}^{2} \sin ^{2} \theta+2 y_{6}^{\prime 2} \cos ^{2} \theta
\end{array}\right) \epsilon_{2}^{2} .
$$

In the following analysis, we will adopt the approximation in eq. (3.23) which results in

$$
M_{1}^{\prime} \sim M_{2}^{\prime} \sim 5 M_{3}^{\prime}, \quad \cos \theta \sim 1, \quad \sin \theta \sim 0.2
$$

In this case, $N_{3}^{\prime}$ is the lightest right-handed neutrino and the CP asymmetry in its decay to the lepton doublet $l_{j}$ can be expressed as [75]

$$
\delta_{3}^{j} \sim \frac{-1}{8 \pi} \frac{1}{\left(\eta \eta^{\dagger}\right)_{33}} \operatorname{Im}\left\{\eta_{1 j} \eta_{3 j}^{*}\left[\frac{3}{2}\left(\eta \eta^{\dagger}\right)_{13} \frac{M_{3}^{\prime}}{M_{1}^{\prime}}+\left(\eta \eta^{\dagger}\right)_{31}\left(\frac{M_{3}^{\prime}}{M_{1}^{\prime}}\right)^{2}\right]\right\} .
$$

The final baryon asymmetry can be estimated by

$$
\frac{n_{\mathrm{B}}}{s}=-c_{s} \sum_{j} \delta_{3}^{j} \kappa
$$

In this equation, $c_{s} \sim 10^{-3}$ is a coefficient accounting for the entropy dilution of $\Delta B$ and conversion factors in the sphaleron process. $\kappa$ which has a value about $10^{-2}-10^{-3}$ is the efficiency factor due to the washout effects. Although there is a CP phase in $\eta$, the total $\mathrm{CP}$ asymmetry is vanishing

$$
\sum_{j} \delta_{3}^{j}=\frac{-1}{8 \pi} \frac{1}{\left(\eta \eta^{\dagger}\right)_{33}} \operatorname{Im}\left\{\left(\eta \eta^{\dagger}\right)_{13}\left[\frac{3}{2}\left(\eta \eta^{\dagger}\right)_{13} \frac{M_{3}^{\prime}}{M_{1}^{\prime}}+\left(\eta \eta^{\dagger}\right)_{31}\left(\frac{M_{3}^{\prime}}{M_{1}^{\prime}}\right)^{2}\right]\right\}=0,
$$

leading to a zero baryon asymmetry.

However, if $N_{3}^{\prime}$ is lighter than $10^{12} \mathrm{GeV}$, the situation will change dramatically. In this case, the Yukawa interaction of $\tau$ will approach equilibrium during the decay process, making $\tau$ distinguishable from $\mu$ and $e$. Thus, we have to take into consideration the flavor 
effects [75] which do not allow the $\mathrm{CP}$ asymmetries for different flavors to be summed directly. Alternatively, every $\delta_{3}^{j}$ should be with a corresponding efficiency factor $\kappa_{j}$ in the expression for baryon asymmetry

$$
\frac{n_{\mathrm{B}}}{s}=-c_{s} \sum_{j} \delta_{3}^{j} \kappa_{j}
$$

where $\kappa_{j}=\kappa / K_{j}$, with $K_{j}$ defined as

$$
K_{j}=\frac{\eta_{3 j} \eta_{3 j}^{*}}{\left(\eta \eta^{\dagger}\right)_{33}}
$$

From the experience of eq. (3.30), we can see that the baryon asymmetry will be proportional to $\left|\kappa_{\tau}-\left(1-\kappa_{\tau}\right)\right|$, which can be approximated as $\kappa \sin ^{2} \theta$. At last, we can get the baryon asymmetry

$$
\frac{n_{\mathrm{B}}}{s} \sim-c_{s} \frac{-3}{16 \pi} y^{2} \epsilon_{2}^{2} \sin \theta \frac{M_{3}^{\prime}}{M_{1}^{\prime}} \kappa \sin ^{2} \theta,
$$

where $y$ is used to denote all the Yukawa couplings and the $\mathcal{O}(1)$ coefficients have been omitted. If we require $M_{3}^{\prime}$ to be in the range $10^{10}-10^{12} \mathrm{GeV}$ and consider that

$$
m_{3} \sim \frac{y^{2} \epsilon_{2}^{2} V_{\mathrm{u}}^{2}}{M_{3}^{\prime}}
$$

$y^{2} \epsilon_{2}^{2}$ should be about $10^{-4}-10^{-2}$. As a consequence, the baryon asymmetry produced will be $10^{-14}-10^{-11}$, consistent with the value given by eq. (3.24).

\subsection{Discussions}

Finally, several comments are given in order.

1. The first thing to note is the NLO corrections might disturb the mixing pattern, so we have to treat them carefully. Due to the setting of this model, there are only two terms contributing to fermion masses at NLO,

$$
y_{7} N_{1}^{c} L H_{\mathrm{u}} \frac{\psi \omega}{\Lambda^{2}}+y_{8} N_{3}^{c} L H_{\mathrm{u}} \frac{\varphi \omega}{\Lambda^{2}} .
$$

After receiving this contribution, the Dirac neutrino mass matrix becomes

$$
M_{\mathrm{D}}^{\prime}=\left(\begin{array}{ccc}
2 y_{4} & -y_{4}-\epsilon_{1} y_{7}^{\prime} & -y_{4}+\epsilon_{3} y_{7}^{\prime} \\
y_{5}^{\prime} & y_{5}^{\prime} & y_{5}^{\prime} \\
2 \epsilon_{3} y_{8} & -y_{6}^{\prime}-\epsilon_{3} y_{8} & y_{6}^{\prime}-\epsilon_{3} y_{8}
\end{array}\right) \epsilon_{2} V_{\mathrm{u}}
$$

where $\epsilon_{3}=V_{5} / \Lambda$ and $y_{7}^{\prime}=y_{7} \sqrt{\left(3 \lambda_{5} \lambda_{17}-3 \lambda_{6} \lambda_{16}\right) /\left(\lambda_{7} \lambda_{17}-\lambda_{6} \lambda_{18}\right)}$. The mass matrix for light neutrinos can be calculated in the usual way

$$
M_{\nu}^{\prime \prime}=M_{\mathrm{D}}^{\prime \mathrm{T}} M_{\mathrm{N}}^{\prime-1} M_{\mathrm{D}}^{\prime}
$$


As before, a TBM rotation is first performed,

$$
\begin{aligned}
& U_{\mathrm{TBM}}^{\mathrm{T}} M_{\nu}^{\prime \prime} U_{\mathrm{TBM}} \\
& =\left(\begin{array}{ccc}
\sqrt{6} y_{4} & & \sqrt{6} \epsilon_{3} y_{8} \\
& \sqrt{3} y_{5}^{\prime} & \\
\sqrt{2} \epsilon_{3} y_{7}^{\prime} & & \sqrt{2} y_{6}^{\prime}
\end{array}\right) M_{\mathrm{N}}^{\prime-1}\left(\begin{array}{ccc}
\sqrt{6} y_{4} & & \sqrt{2} \epsilon_{3} y_{7}^{\prime} \\
& \sqrt{3} y_{5}^{\prime} & \\
\sqrt{6} \epsilon_{3} y_{8} & & \sqrt{2} y_{6}^{\prime}
\end{array}\right)\left(\epsilon_{2} V_{\mathrm{u}}\right)^{2} \\
& =\left(\begin{array}{cc}
\frac{6\left(\epsilon_{3}^{2} y_{8}^{2} M_{1}-2 \epsilon_{3} y_{4} y_{8} \Delta M+y_{4}^{2} M_{3}\right)}{D} & \frac{2 \sqrt{3}\left(\epsilon_{3} y_{6}^{\prime} y_{8} M_{1}-y_{4} y_{6}^{\prime} \Delta M+\epsilon_{3} y_{4} y_{7}^{\prime} M_{3}\right)}{D} \\
\cdots & \frac{3 y_{5}^{\prime 2}}{M_{2}} \\
\cdots & \frac{2\left(y_{6}^{\prime 2} M_{1}-2 \epsilon_{3} y_{6}^{\prime} y_{7}^{\prime} \Delta M+\epsilon_{3}^{2} y_{7}^{\prime 2} M_{3}\right)}{D}
\end{array}\right)\left(\epsilon_{2} V_{\mathrm{u}}\right)^{2} .
\end{aligned}
$$

This matrix is then diagonalized by $\mathrm{U}\left(\theta_{13}^{\prime}\right)$ with

$$
\begin{aligned}
& \rho=0, \quad \tan 2 \theta_{13}^{\prime}=\frac{2 \sqrt{3}\left(\epsilon_{3} y_{6}^{\prime} y_{8} M_{1}-y_{4} y_{6}^{\prime} \Delta M+\epsilon_{3} y_{4} y_{7}^{\prime} M_{3}\right)}{y_{6}^{\prime 2} M_{1}+2 \epsilon_{3}\left(3 y_{4} y_{8}-y_{6}^{\prime} y_{7}^{\prime}\right) \Delta M-3 y_{4}^{2} M_{3}}, \quad \text { if } \quad V_{5}= \pm \frac{M}{\sqrt{\lambda_{4}}} \\
& \rho=\frac{\pi}{2} / \frac{3 \pi}{2}, \quad \tan 2 \theta_{13}^{\prime}=\frac{2 \sqrt{3}\left(\left|\epsilon_{3}\right| y_{6}^{\prime} y_{8} M_{1}-y_{4} y_{6}^{\prime}|\Delta M|+\left|\epsilon_{3}\right| y_{4} y_{7}^{\prime} M_{3}\right)}{y_{6}^{\prime 2} M_{1}-2 \epsilon_{3}\left(3 y_{4} y_{8}+y_{6}^{\prime} y_{7}^{\prime}\right) \Delta M+3 y_{4}^{2} M_{3}}, \quad \text { if } \quad V_{5}= \pm i \frac{M}{\sqrt{\lambda_{4}}} .
\end{aligned}
$$

Thus, we can say the mixing pattern is stable against at least NLO corrections.

2. If the requirement of $\mathrm{CP}$ symmetry is relaxed, this model can give the TM2 mixing with an arbitrary $\rho$, which is determined by the diagonalization of eq. (3.20) in which $y_{4}, y_{5}^{\prime}$ and $y_{6}^{\prime}$ are complex coefficients for now. In this case, $\theta_{23}$ can be obtained through eq. (2.17). For example, if we take $\rho$ (which approximates to $\delta$ in the standard parameterization) as the best-fit value $1.34 \pi$ in table $1, \sin ^{2} \theta_{23}$ will be 0.55 and close to the best-fit value 0.567 .

3. Analogously, the model building and phenomenological analysis in this section completely apply to the TM1 mixing [76-78]

$$
U_{\mathrm{PMNS}}=\left(\begin{array}{ccc}
\frac{\sqrt{2}}{\sqrt{3}} & \frac{1}{\sqrt{3}} & 0 \\
-\frac{1}{\sqrt{6}} & \frac{1}{\sqrt{3}} & \frac{1}{\sqrt{2}} \\
\frac{1}{\sqrt{6}} & -\frac{1}{\sqrt{3}} & \frac{1}{\sqrt{2}}
\end{array}\right)\left(\begin{array}{ccc}
1 & & \\
\cos \theta_{23}^{\prime} & \sin \theta_{23}^{\prime} e^{-i \rho} \\
-\sin \theta_{23}^{\prime} e^{i \rho} & \cos \theta_{23}^{\prime}
\end{array}\right) .
$$

Put simply, if we assign $\xi$ the quantum numbers of $Z_{2}^{2}$ and $Z_{2}^{3}$, its VEV will introduce the mixing between $N_{2}$ and $N_{3}$. That is to say, the PMNS matrix would be the TBM matrix multiplied by a 2-3 rotation from the right-hand side, just like the TM1 mixing.

4. The last point to stress is that the CP symmetry imposed on the model is consistent with the flavor symmetry $A 4$, although it is not defined in the way as the so-called generalized CP transformation does. This is because in the particular basis of $A 4$ we have chosen [58], the would-be generalized CP transformation can be represented as the identity matrix times a phase, i.e., a trivial one [79]. In other words, the naive CP transformation $\phi \rightarrow \phi^{*}$ can work well in our model. 


\section{Summary}

In this paper, we have attempted to modify the TBM mixing as minimally as possible to accommodate the recent observation of a relatively large $\theta_{13}$. Above all, we have examined whether there is a neutrino mass matrix with a simple form that can describe the realistic mixing scheme. By analogy with that for the TBM mixing, we find one neutrino mass matrix with only three independent parameters, which is connected with the FriedbergLee symmetry and $\mu-\tau$ symmetry breaking. Unlike in the TBM case, the masses and mixing angles of this mass matrix are correlated, so that the mass values can be determined with reference to the values of mixing angles. The values of mass sum and $m_{\beta \beta}$ are close to the experimental sensitivities, thus will be observed or excluded in a short time.

As a matter of fact, the mixing pattern given by this mass matrix is the so-called TM2 mixing. Therefore, we have also discussed the way to build a model generating this mixing pattern, invoking the minimal modification to a model that produces the TBM mixing [80]. The model is constructed with $A_{4} \times \mathrm{U}(1) \times Z_{2}^{1} \times Z_{2}^{2} \times Z_{2}^{3}$ as the flavor symmetries. The mass matrix for charged leptons is diagonal and the hierarchies among them are guaranteed by the U(1) symmetry. Similarly, the mass matrix for right-handed neutrinos is also diagonal, because of the $Z_{2}^{1} \times Z_{2}^{2} \times Z_{2}^{3}$ symmetry. Their Yukawa couplings with left-handed neutrinos have a special form, as a consequence of the specific VEV alignments, which are justified to NLO, possessed by the flavon fields. At this stage, the mixing pattern for light neutrinos is the TBM after the seesaw mechanism. However, a flavon field which acquires a VEV can introduce the mixing between the first and third right-handed neutrinos, transforming the mixing pattern to the TM2. More interestingly, this VEV can be purely imaginary if we impose the CP symmetry on this model, leading to the maximal CP violation and maximal $\theta_{23}$. On the other side, this maximal $\mathrm{CP}$ violation gives a zero total $\mathrm{CP}$ asymmetry in the leptogenesis mechanism. But after the flavor effects are considered, the observed value of baryon asymmetry can be marginally reproduced. If the CP symmetry is given up, the CP phase will become free and $\theta_{23}$ can departure from $45^{\circ}$. Besides, it is found the NLO contributions do not change the mixing pattern. The last thing to mention is that this approach can be directly generalized to realizing the TM1 mixing.

In conclusion, the TBM mixing can be modified in a minimal way, in terms of both the mass matrix and model building, to accommodate the non-zero $\theta_{13}$ and $\mathrm{CP}$ phase. Therefore, it can still serve as a guide for model building.

\section{Acknowledgments}

I am very grateful to Professor Zhi-zhong Xing for useful comments and suggestions and Professor Chun Liu for his support. I am also indebted to Jue Zhang and Shun Zhou for their help with the flavor effects in leptogenesis and Ye-ling Zhou for helpful discussions about generalized CP transformation. This work was supported in part by the National Natural Science Foundation of China under Nos. 11375248 and 11135009.

Open Access. This article is distributed under the terms of the Creative Commons Attribution License (CC-BY 4.0), which permits any use, distribution and reproduction in any medium, provided the original author(s) and source are credited. 


\section{References}

[1] B. Pontecorvo, Neutrino Experiments and the Problem of Conservation of Leptonic Charge, Sov. Phys. JETP 26 (1968) 984 [Zh. Eksp. Teor. Fiz. 53 (1967) 1717] [InSPIRE].

[2] Z. Maki, M. Nakagawa and S. Sakata, Remarks on the unified model of elementary particles, Prog. Theor. Phys. 28 (1962) 870 [inSPIRE].

[3] N. Cabibbo, Unitary Symmetry and Leptonic Decays, Phys. Rev. Lett. 10 (1963) 531 [INSPIRE].

[4] M. Kobayashi and T. Maskawa, CP Violation in the Renormalizable Theory of Weak Interaction, Prog. Theor. Phys. 49 (1973) 652 [inSPIRE].

[5] R. Davis Jr., D.S. Harmer and K.C. Hoffman, Search for neutrinos from the sun, Phys. Rev. Lett. 20 (1968) 1205 [InSPIRE].

[6] Super-Kamiokande collaboration, Y. Fukuda et al., Evidence for oscillation of atmospheric neutrinos, Phys. Rev. Lett. 81 (1998) 1562 [hep-ex/9807003] [INSPIRE].

[7] DAYA-BAY collaboration, F.P. An et al., Observation of electron-antineutrino disappearance at Daya Bay, Phys. Rev. Lett. 108 (2012) 171803 [arXiv:1203.1669] [INSPIRE].

[8] MINOS collaboration, P. Adamson et al., Measurement of Neutrino and Antineutrino Oscillations Using Beam and Atmospheric Data in MINOS, Phys. Rev. Lett. 110 (2013) 251801 [arXiv:1304.6335] [INSPIRE].

[9] D.V. Forero, M. Tortola and J.W.F. Valle, Neutrino oscillations refitted, arXiv:1405.7540 [INSPIRE].

[10] P.F. Harrison, D.H. Perkins and W.G. Scott, Tri-bimaximal mixing and the neutrino oscillation data, Phys. Lett. B 530 (2002) 167 [hep-ph/0202074] [INSPIRE].

[11] Z.-z. Xing, Nearly tri bimaximal neutrino mixing and CP-violation, Phys. Lett. B 533 (2002) 85 [hep-ph/0204049] [INSPIRE].

[12] E. Ma and G. Rajasekaran, Softly broken $A_{4}$ symmetry for nearly degenerate neutrino masses, Phys. Rev. D 64 (2001) 113012 [hep-ph/0106291] [INSPIRE].

[13] G. Altarelli and F. Feruglio, Tri-bimaximal neutrino mixing from discrete symmetry in extra dimensions, Nucl. Phys. B 720 (2005) 64 [hep-ph/0504165] [INSPIRE].

[14] G. Altarelli, F. Feruglio and L. Merlo, Tri-Bimaximal Neutrino Mixing and Discrete Flavour Symmetries, Fortsch. Phys. 61 (2013) 507 [arXiv:1205.5133] [InSPIRE].

[15] C.H. Albright, A. Dueck and W. Rodejohann, Possible Alternatives to Tri-bimaximal Mixing, Eur. Phys. J. C 70 (2010) 1099 [arXiv:1004.2798] [INSPIRE].

[16] S.F. King, Tri-bimaximal Neutrino Mixing and $\theta_{13}$, Phys. Lett. B 675 (2009) 347 [arXiv:0903.3199] [INSPIRE].

[17] Y. Lin, Tri-bimaximal Neutrino Mixing from $A_{4}$ and $\theta_{13} \sim \theta C$, Nucl. Phys. B 824 (2010) 95 [arXiv:0905.3534] [INSPIRE].

[18] X.-G. He and A. Zee, Minimal Modification to Tri-bimaximal Mixing, Phys. Rev. D 84 (2011) 053004 [arXiv: 1106.4359] [InSPIRE]. 
[19] S. Morisi, K.M. Patel and E. Peinado, Model for T2K indication with maximal atmospheric angle and tri-maximal solar angle, Phys. Rev. D 84 (2011) 053002 [arXiv:1107.0696] [INSPIRE].

[20] Y.H. Ahn, H.-Y. Cheng and S. Oh, An extension of tribimaximal lepton mixing, Phys. Rev. D 84 (2011) 113007 [arXiv:1107.4549] [InSPIRE].

[21] S.F. King and C. Luhn, $A_{4}$ models of tri-bimaximal-reactor mixing, JHEP 03 (2012) 036 [arXiv:1112.1959] [INSPIRE].

[22] T. Kitabayashi, A way of the minimal modification to tribimaximal mixing, Mod. Phys. Lett. A 27 (2012) 1250180 [InSPIRE].

[23] W. Rodejohann and H. Zhang, Simple two Parameter Description of Lepton Mixing, Phys. Rev. D 86 (2012) 093008 [arXiv: 1207.1225] [INSPIRE].

[24] B. Hu, Trimaximal-Cabibbo neutrino mixing: A parametrization in terms of deviations from tribimaximal mixing, Phys. Rev. D 87 (2013) 053011 [arXiv:1212.4079] [INSPIRE].

[25] W. Chao and Y.-j. Zheng, Relatively Large Theta13 from Modification to the Tri-bimaximal, Bimaximal and Democratic Neutrino Mixing Matrices, JHEP 02 (2013) 044 [arXiv: 1107.0738] [INSPIRE].

[26] A. Rashed, Deviation from Tri-Bimaximal Mixing and Large Reactor Mixing Angle, Nucl. Phys. B 874 (2013) 679 [arXiv:1111.3072] [INSPIRE].

[27] V.V. Vien and H.N. Long, The $D_{4}$ flavor symmetry in 3-3-1 model with neutral leptons, Int. J. Mod. Phys. A 28 (2013) 1350159.

[28] W. Grimus and P.O. Ludl, Two-parameter neutrino mass matrices with two texture zeros, J. Phys. G 40 (2013) 055003 [arXiv: 1208.4515] [INSPIRE].

[29] S. Chakdar, K. Ghosh and S. Nandi, A model for Dirac neutrino mass matrix with only four parameters, arXiv:1405.2328 [INSPIRE].

[30] H. Nishiura and T. Fukuyama, Simple neutrino mass matrix with only two free parameters, arXiv: 1405.2416 [INSPIRE].

[31] Z.-z. Xing and S. Zhou, Tri-bimaximal Neutrino Mixing and Flavor-dependent Resonant Leptogenesis, Phys. Lett. B 653 (2007) 278 [hep-ph/0607302] [INSPIRE].

[32] R. Friedberg and T.D. Lee, A Possible Relation between the Neutrino Mass Matrix and the Neutrino Mapping Matrix, High Energy Phys. Nuclear Phys. 30 (2006) 591 [hep-ph/0606071] [INSPIRE].

[33] Z.-z. Xing, H. Zhang and S. Zhou, Nearly Tri-bimaximal Neutrino Mixing and CP-violation from $\mu-\tau$ Symmetry Breaking, Phys. Lett. B 641 (2006) 189 [hep-ph/0607091] [INSPIRE].

[34] T. Araki and R. Takahashi, Tri-Bimaximal Mixing from Twisted Friedberg-Lee Symmetry, Eur. Phys. J. C 63 (2009) 521 [arXiv:0811.0905] [inSPIRE].

[35] N. Razzaghi and S.S. Gousheh, Generalized Friedberg-Lee model for CP-violation in neutrino physics, Phys. Rev. D 86 (2012) 053006 [arXiv:1211.4389] [InSPIRE].

[36] R.N. Mohapatra, $\theta_{13}$ as a probe of $\mu \leftrightarrow \tau$ symmetry for leptons, JHEP 10 (2004) 027 [hep-ph/0408187] [INSPIRE].

[37] R.N. Mohapatra and W. Rodejohann, Broken $\mu-\tau$ symmetry and leptonic CP-violation, Phys. Rev. D 72 (2005) 053001 [hep-ph/0507312] [INSPIRE]. 
[38] I. Aizawa and M. Yasue, A New type of complex neutrino mass texture and $\mu-\tau$ symmetry, Phys. Rev. D 73 (2006) 015002 [hep-ph/0510132] [InSPIRE].

[39] T. Baba and M. Yasue, Correlation between Leptonic CP-violation and $\mu-\tau$ Symmetry Breaking, Phys. Rev. D 75 (2007) 055001 [hep-ph/0612034] [InSPIRE].

[40] C.H. Albright and W. Rodejohann, Comparing Trimaximal Mixing and Its Variants with Deviations from Tri-bimaximal Mixing, Eur. Phys. J. C 62 (2009) 599 [arXiv:0812.0436] [INSPIRE].

[41] H.-J. He and F.-R. Yin, Common Origin of $\mu-\tau$ and CP Breaking in Neutrino Seesaw, Baryon Asymmetry and Hidden Flavor Symmetry, Phys. Rev. D 84 (2011) 033009 [arXiv: 1104.2654] [INSPIRE].

[42] Z.-z. Xing, A Further study of $\mu-\tau$ symmetry breaking at neutrino telescopes after the Daya Bay and RENO measurements of $\theta_{13}$, Phys. Lett. B 716 (2012) 220 [arXiv:1205.6532] [INSPIRE].

[43] J.J. Liao, D. Marfatia and K. Whisnant, Perturbations to $\mu-\tau$ symmetry in neutrino mixing, Phys. Rev. D 87 (2013) 013003 [arXiv:1205.6860] [INSPIRE].

[44] A. Damanik, Nonzero $\theta_{13}, C P$-violation and $\mu-\tau$ Symmetry, arXiv:1305.6900 [INSPIRE].

[45] K. Fuki and M. Yasue, What does $\mu-\tau$ symmetry imply in neutrino mixings?, Phys. Rev. D 73 (2006) 055014 [hep-ph/0601118] [INSPIRE].

[46] T2K collaboration, K. Abe et al., Precise Measurement of the Neutrino Mixing Parameter $\theta_{23}$ from Muon Neutrino Disappearance in an Off-axis Beam, Phys. Rev. Lett. 112 (2014) 181801 [arXiv:1403.1532] [INSPIRE].

[47] MINOS collaboration, P. Adamson et al., Combined analysis of $\nu_{\mu}$ disappearance and $\nu_{\mu} \rightarrow \nu_{e}$ appearance in MINOS using accelerator and atmospheric neutrinos,

Phys. Rev. Lett. 112 (2014) 191801 [arXiv:1403.0867] [INSPIRE].

[48] P.F. Harrison and W.G. Scott, Symmetries and generalizations of tri-bimaximal neutrino mixing, Phys. Lett. B 535 (2002) 163 [hep-ph/0203209] [INSPIRE].

[49] P.F. Harrison and W.G. Scott, $\mu-\tau$ reflection symmetry in lepton mixing and neutrino oscillations, Phys. Lett. B 547 (2002) 219 [hep-ph/0210197] [INSPIRE].

[50] R. Krishnan, P.F. Harrison and W.G. Scott, Simplest Neutrino Mixing from $S_{4}$ Symmetry, JHEP 04 (2013) 087 [arXiv:1211.2000] [InSPIRE].

[51] C. Jarlskog, Commutator of the Quark Mass Matrices in the Standard Electroweak Model and a Measure of Maximal CP-violation, Phys. Rev. Lett. 55 (1985) 1039 [INSPIRE].

[52] S. Gupta, A.S. Joshipura and K.M. Patel, Minimal extension of tri-bimaximal mixing and generalized $Z_{2} \times Z_{2}$ symmetries, Phys. Rev. D 85 (2012) 031903 [arXiv:1112.6113] [INSPIRE].

[53] P.M. Ferreira, W. Grimus, L. Lavoura and P.O. Ludl, Maximal CP-violation in Lepton Mixing from a Model with $\Delta(27)$ flavour Symmetry, JHEP 09 (2012) 128 [arXiv: 1206.7072] [INSPIRE].

[54] N. Memenga, W. Rodejohann and H. Zhang, $A_{4}$ flavor symmetry model for Dirac neutrinos and sizable $U_{e 3}$, Phys. Rev. D 87 (2013) 053021 [arXiv: 1301.2963] [INSPIRE].

[55] S.F. King, T. Neder and A.J. Stuart, Lepton mixing predictions from $\Delta\left(6 n^{2}\right)$ family Symmetry, Phys. Lett. B 726 (2013) 312 [arXiv:1305.3200] [INSPIRE]. 
[56] G.-J. Ding, S.F. King and A.J. Stuart, Generalised CP and $A_{4}$ Family Symmetry, JHEP 12 (2013) 006 [arXiv: 1307.4212] [INSPIRE].

[57] F. Feruglio, C. Hagedorn and R. Ziegler, A realistic pattern of lepton mixing and masses from $S_{4}$ and $C P$, Eur. Phys. J. C 74 (2014) 2753 [arXiv:1303.7178] [INSPIRE].

[58] G. Altarelli and F. Feruglio, Tri-bimaximal neutrino mixing, $A_{4}$ and the modular symmetry, Nucl. Phys. B 741 (2006) 215 [hep-ph/0512103] [INSPIRE].

[59] S.F. King and C. Luhn, Neutrino Mass and Mixing with Discrete Symmetry, Rept. Prog. Phys. 76 (2013) 056201 [arXiv:1301.1340] [InSPIRE].

[60] S.F. King, A. Merle, S. Morisi, Y. Shimizu and M. Tanimoto, Neutrino Mass and Mixing: from Theory to Experiment, New J. Phys. 16 (2014) 045018 [arXiv:1402.4271] [InSPIRE].

[61] P. Minkowski, $\mu \rightarrow$ ey at a Rate of One Out of $10^{9}$ Muon Decays?, Phys. Lett. B 67 (1977) 421 [INSPIRE].

[62] M. Gell-Mann, P. Ramond and R. Slansky, in Supergravity, P. van Nieuwenhuizen and D. Freedman eds., North-Holland (1979), pg. 315.

[63] T. Yanagida, Horizontal Symmetry and Masses of Neutrinos, Prog. Theor. Phys. 64 (1980) 1103 [inSPIRE].

[64] R.N. Mohapatra and G. Senjanović, Neutrino Mass and Spontaneous Parity Violation, Phys. Rev. Lett. 44 (1980) 912 [InSPIRE].

[65] G.C. Branco, R. Gonzalez Felipe, F.R. Joaquim and H. Serodio, Spontaneous leptonic CP-violation and nonzero $\theta_{13}$, Phys. Rev. D 86 (2012) 076008 [arXiv:1203.2646] [INSPIRE].

[66] G.-J. Ding, S.F. King, C. Luhn and A.J. Stuart, Spontaneous CP-violation from vacuum alignment in $S_{4}$ models of leptons, JHEP 05 (2013) 084 [arXiv: 1303.6180] [INSPIRE].

[67] F. Feruglio, C. Hagedorn and R. Ziegler, Lepton Mixing Parameters from Discrete and CP Symmetries, JHEP 07 (2013) 027 [arXiv:1211.5560] [INSPIRE].

[68] S. Antusch, S.F. King and M. Spinrath, Spontaneous CP-violation in $A_{4} \times \mathrm{SU}(5)$ with Constrained Sequential Dominance 2, Phys. Rev. D 87 (2013) 096018 [arXiv:1301.6764] [INSPIRE].

[69] Y.H. Ahn, S.K. Kang and C.S. Kim, Spontaneous CP-violation in $A_{4}$ Flavor Symmetry and Leptogenesis, Phys. Rev. D 87 (2013) 113012 [arXiv: 1304.0921] [INSPIRE].

[70] G.-J. Ding and Y.-L. Zhou, Lepton mixing parameters from $\Delta(48)$ family symmetry and generalised CP, JHEP 06 (2014) 023 [arXiv:1404.0592] [INSPIRE].

[71] C.D. Froggatt and H.B. Nielsen, Hierarchy of Quark Masses, Cabibbo Angles and CP-violation, Nucl. Phys. B 147 (1979) 277 [inSPIRE].

[72] S. Antusch, S.F. King, C. Luhn and M. Spinrath, Right Unitarity Triangles and Tri-Bimaximal Mixing from Discrete Symmetries and Unification, Nucl. Phys. B 850 (2011) 477 [arXiv:1103.5930] [INSPIRE].

[73] M. Fukugita and T. Yanagida, Baryogenesis Without Grand Unification, Phys. Lett. B 174 (1986) 45 [INSPIRE].

[74] F.R. Klinkhamer and N.S. Manton, A Saddle Point Solution in the Weinberg-Salam Theory, Phys. Rev. D 30 (1984) 2212 [INSPIRE]. 
[75] E. Nardi, Y. Nir, E. Roulet and J. Racker, The Importance of flavor in leptogenesis, JHEP 01 (2006) 164 [hep-ph/0601084] [INSPIRE].

[76] I. de Medeiros Varzielas and L. Lavoura, Flavour models for TM $M_{1}$ lepton mixing, J. Phys. G 40 (2013) 085002 [arXiv:1212.3247] [INSPIRE].

[77] C. Luhn, Trimaximal $T M_{1}$ neutrino mixing in $S_{4}$ with spontaneous CP-violation, Nucl. Phys. B 875 (2013) 80 [arXiv:1306.2358] [INSPIRE].

[78] C.-C. Li and G.-J. Ding, Generalised CP and trimaximal $T M_{1}$ lepton mixing in $S_{4}$ family symmetry, Nucl. Phys. B $\mathbf{8 8 1}$ (2014) 206 [arXiv:1312.4401] [INSPIRE].

[79] M. Holthausen, M. Lindner and M.A. Schmidt, CP and Discrete Flavour Symmetries, JHEP 04 (2013) 122 [arXiv: 1211.6953] [INSPIRE].

[80] B. Karmakar and A. Sil, Nonzero $\theta_{13}$ and Leptogenesis in a Type-I See-saw Model with $A_{4}$ Symmetry, arXiv:1407.5826 [INSPIRE]. 